\title{
Derivation of Equations for a Size Distribution of Spherical Particles in Non-Transparent Materials
}

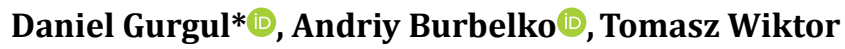 \\ AGH University of Science and Technology, Faculty of Foundry Engineering, 23 Reymonta St., 30-059 Krakow, Poland \\ *e-mail: dg@agh.edu.pl
}

(C) 2021 Authors. This is an open access publication, which can be used, distributed and reproduced in any medium according to the Creative Commons CC-BY 4.0 License requiring that the original work has been properly cited.

Received: 25 May 2021/ Accepted: 9 September 2021/ Published online: 20 September 2021.

This article is published with open access at AGH University of Science and Technology Press.

\begin{abstract}
This paper presents a new proposition on how to derive mathematical formulas that describe an unknown Probability Density Function $\left(\mathrm{PDF}_{3}\right)$ of the spherical radii $\left(r_{3}\right)$ of particles randomly placed in non-transparent materials. We have presented two attempts here, both of which are based on data collected from a random planar cross-section passed through space containing three-dimensional nodules. The first attempt uses a Probability Density Function $\left(\mathrm{PDF}_{2}\right)$ the form of which is experimentally obtained on the basis of a set containing two-dimensional radii $\left(r_{2}\right)$. These radii are produced by an intersection of the space by a random plane. In turn, the second solution also uses an experimentally obtained Probability Density Function $\left(\mathrm{PDF}_{1}\right)$. But the form of $\mathrm{PDF}_{1}$ has been created on the basis of a set containing chord lengths collected from a cross-section. The most important finding presented in this paper is the conclusion that if the $\mathrm{PDF}_{1}$ has proportional scopes, the PDF $\mathrm{must}_{3}$ have a constant value in these scopes. This fact allows stating that there are no nodules in the sample space that have particular radii belonging to the proportional ranges the $\mathrm{PDF}_{1}$.
\end{abstract}

\section{Keywords:}

planimetric analysis, linear analysis, estimation of diameter sizes distribution, probability density function

\section{INTRODUCTION}

The problem of mapping an unknown probability distribution of spherical particles size, randomly placed in non-transparent materials, is one of the classical stereological tasks. The data (a set of mark radii, an area of intersections or chord lengths) used in such an analysis, is obtained from an examination of flat cross-sections (random cutting planes). Doing such research, it is important to be aware of two facts:

1. generally, a random cutting plane intersects three-dimensional particles outside their centre points. Hence, a mean value of mark radii, an area of intersections or chord lengths differs from the mean value of the linear sizes of nodules,

2. a random cutting plane intersects bigger particles more often than smaller ones, rather than this results from the true count of the particles.

To solve this stereological problem, many attempts have been proposed and those solutions can be categorised into three groups. The first group includes methods that use mark radii as input data [1-3]. The second group uses areas of intersected nodules [4]. The last one employs a set of random chords [5-7].

The methods from the first group are currently most frequently used for an analysis of the graphite size distribution in ductile iron [8-10]. The accuracy of these methods has been improved in [11].

Our paper develops the method from the third group. New aspects which distinguish our work from others are different form of a constant parameter in the final equation and the statement of the fact that if $\mathrm{PDF}_{1}$ has proportional ranges, the sample space does not have nodules with a particular size belonging to these proportional scopes.

This article is also an extension of our previous work [12] which deals with the same problem. However, the information included there is too concise and required an extension and unification of variables and symbols. We decided here to add more explanations and figures that help understand the derivation of the formulas for readers who are unfamiliar with probability theory and mathematical statistics. We have also proposed another way for the estimation of the expected value of the outer particles' area.

A list of variables used in the text can be found at the end of the article. 


\section{MODEL ASSUMPTIONS}

Let us consider sample space (Fig. 1) in which spherical particles, with an unknown Probability Density Function $\left(\mathrm{PDF}_{3}\right)$ of particles' radii $\left(r_{3}\right)$, are randomly placed. Let us assume that we insert into the space a random cutting plane or a random secant. Both, the cutting plane and the secant leave marks on the surfaces of the intersected particles. The cutting plane leaves circular marks (the black circles in Fig ure 1) with radii denoted $r_{2}$. In turn, when the secant pierces the nodules, it becomes divided by the nodules' surface, into fragments. The secant's fragments (the blue segments in Figure 1) lying inside particles will be called chords and their half-length denoted $r_{1}$.

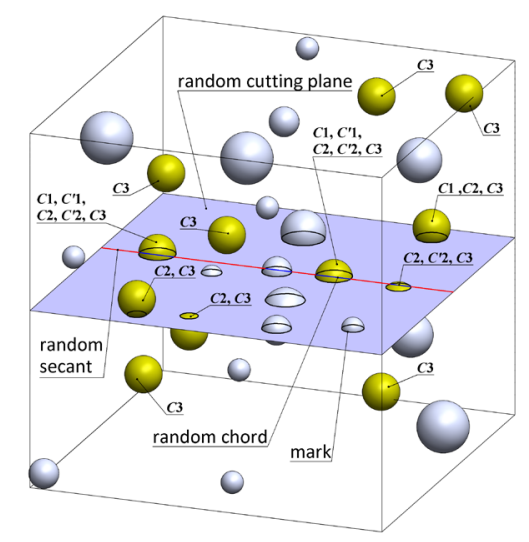

Fig. 1. Sample space with randomly placed particles with random radii. The meaning of the symbols is explained further in the paper

We now want to estimate the form of $\mathrm{PDF}_{3}$ having only a set of radii $r_{2}$ or chords $r_{1}$. To derive the mathematical formulas which solve this problem we must impose a few assumptions on our sample space:

- the space contains nodules the count of which is determined by a volumetric particle density equal to $N_{3}$ (a mean nodule count per unit volume),

- the nodule centres count in a selected volume is proportional to the size of this volume,

- the likelihood of intersection between nodules is so small that it can be neglected,

- the radii's size of the particles is described by an unknown continuous Probability Density Function ( $\mathrm{PDF}_{3}$ ), or by an unknown continuous Cumulative Distribution Function $\left(\mathrm{CDF}_{3}\right)$,

- the radius of the largest nodule does not exceed the value of $R_{\max }$.

As a consequence of the previous assumptions, we claim the following facts for a particle intersected by a random plane (see Fig. 2 in next section):

- the variable $h_{2}$ which is the distance between the nodule's centre and the cutting plane, has a uniform continuous distribution,

- similarly, the distance $h_{1}$ between the random chord and the nodule's centre has also a uniform continuous distribution.
Before we proceed further, and in order to help the reader understand the derivation process, we want to recall and add a short description of the basic properties of two fundamental probability calculus functions. These functions are defined on a single real-valued continuous random variable $X$. We would like to emphasise that the information provided by us is not a strictly formal mathematical definition. We have omitted many things in order to use only the ones that will be necessary for our needs.

The first function is a Probability Density Function (PDF). The PDF, denoted $f_{X}(x)$, is non-negative and continuous on its domain. The domain of this function is a set of real numbers from $-\infty$ to $+\infty$. The formula of the PDF allows us to calculate the likelihood of occurrence of $X$ in the range between $a$ and $b$. This is written as the following integral:

$$
\operatorname{Pr}(a<X \leq b)=\int_{a}^{b} f_{X}(x) \mathrm{d} x
$$

where $x$ is any real-valued number.

The second function which we want to recall here is a Cumulative Distribution Function (CDF) denoted as $F_{X}(x)$. The CDF is non-decreasing and continuous. Its domain and range are sets from $-\infty$ to $+\infty$ and from 0 to 1 , respectively. The result of $F_{X}(x)$ is the probability that the random variable $X$ takes on a value less than or equal to $x$. This is written as:

$$
F_{X}(x)=\operatorname{Pr}(X \leq x)
$$

The fundamental parameter of any random variable is the Expected Value denoted $E[X]$. It is calculated as the integral:

$$
E[X]=\int_{-\infty}^{+\infty} x f_{X}(x) \mathrm{d} x
$$

According to probability theory two important facts result from Equations (1) and (2):

$$
\begin{gathered}
\operatorname{Pr}(-\infty<X<+\infty)=\int_{-\infty}^{+\infty} f_{X}(x) \mathrm{d} x=1 \\
\operatorname{Pr}(a<X \leq b)=F_{X}(b)-F_{X}(a)
\end{gathered}
$$

The PDF can be determined from the CDF by differentiating using the Fundamental Theorem of Calculus:

$$
f_{X}(x)=\frac{\mathrm{d} F_{X}(x)}{\mathrm{d} x}
$$

Another important dependency between mentioned functions is:

$$
F_{X}(x)=\int_{-\infty}^{x} f_{X}(t) \mathrm{d} t
$$

The last thing from the probability theory that we want to remind and which we will use in the text is the so-called 
Geometric Probability. Suppose that we have space $\Omega$ in which there is subspace $A$, where $A \subseteq \Omega$. We want now to calculate the likelihood of an event $A$ that a randomly placed point in the space $\Omega$ will also be included in subspace $A$. To answer this question, we can use the following simple formula:

$$
\operatorname{Pr}(A)=\frac{|A|}{|\Omega|}
$$

where $|A|$ and $|\Omega|$ is a measure of the space $A$ and $\Omega$, respectively.

Let us now adapt Equation (2) to the random variable $r_{1}, r_{2}$ and $r_{3}$ depicted in Figures 1 and 2 . The fraction of all particles, with the radii meeting the inequality $r_{3} \leq x$, is determined by the $\mathrm{CDF}_{3}$ - a function of $F_{3}(x)$. Similarly, we can determine, by $\mathrm{CDF}_{2}$ - a function of $F_{2}(x)$ - the fraction of all nodules having their marks radius $r_{2} \leq x$. Finally, the $\mathrm{CDF}_{1}$ - a function of $F_{1}(x)$ - describes the fraction of all the particles that have a half-length chord meeting the inequality $r_{1} \leq x$.

It is obvious that the largest mark radius $r_{2}$, as well as the largest half-chord $r_{1}$ cannot be greater than the value of $R_{\max }$. Therefore, taking into account Equations (2) and (6) we can define the following function for our needs:

$$
f_{i}(x)=\left\{\begin{array}{ccc}
0 & x \leq 0 \\
\mathrm{~d} F_{i}(x) / \mathrm{d} x & \text { for } & 0<x \leq R_{\max } \\
0 & & x>R_{\max }
\end{array}\right.
$$

where the subscript $i$ can take one of the values of 1, 2, 3, that means a half-length chord, a mark radius and a particle radius, respectively. Similarly, the variable $x$ can be substituted by $r_{1}, r_{2}$ or $r_{3}$ depending on our need.

Our derivation of the formulas estimating the form of the $\mathrm{PDF}_{3}$ is divided into two parts. In the first part, named Planimetric Analysis, we will derive equations, taking into account mark radii $r_{2}$. In turn, in the second part, named Linear Analysis, formulas will be derived using half chord lengths $r_{1}$.

\section{PLANIMETRIC ANALYSIS}

Between the particle's radius $r_{3}$ and the mark's radius $r_{2}$ (Fig. 2) there is an obvious relation $r_{3}^{2}=r_{2}^{2}+h_{2}^{2}$. Therefore, getting a nodule section with the mark's radius $r_{2} \geq x$ requires meeting two conditions:

$$
\begin{gathered}
r_{3} \geq x \\
h_{2} \leq \sqrt{r_{3}^{2}-x^{2}}
\end{gathered}
$$

The probability of meeting the condition (11) can be calculated by using the definition from Equation (8):

$$
\operatorname{Pr}\left(h_{2} \leq \sqrt{r_{3}^{2}-x^{2}}\right)=\frac{\sqrt{r_{3}^{2}-x^{2}}}{r_{3}}
$$

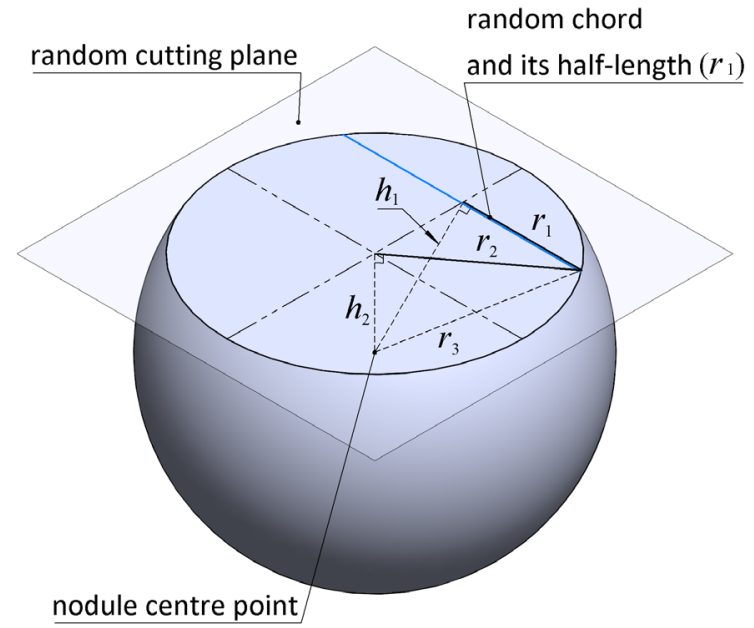

Fig. 2. A spherical particle intersected by a random plane. The symbols have the following meaning: $r_{1}$ - half-length of the random chord; $r_{2}$ - mark radius; $r_{3}$ - nodule radius; $h_{1}$ - distance between the random chord and the nodule centre; $h_{2}$ - distance between the random cutting plane and the nodule centre

Let us now pick out, from a unit volume of the sample space seen in Figure 1, only those particles that have their radius in the range from $r_{3}$ to $r_{3}+\mathrm{d} r_{3}$. We will name this group a class or a set and denote it $C 3$. The quantity $\mathrm{d} n_{C 3}$ of the nodules belonging to the $C 3$ can be estimated by using the $\mathrm{PDF}_{3}$ :

$$
\mathrm{d} n_{C 3}\left(r_{3}\right)=N_{3} f_{3}\left(r_{3}\right) \mathrm{d} r_{3}
$$

where $N_{3}$ is a mean nodule count per unit volume.

Among the class $C 3$, the random cutting plane only intersects these particles which have their centre points on both sides of this plane, offset from the plane at a distance of less than the radius $r_{3}$. This new subclass will be denoted $C 2$. The quantity $\mathrm{d} n_{C 2}$, per unit area of the cutting plane, of all the nodules belonging to the $C 2$, may be calculated by multiplying Equation (13) by doubled distance $\left(2 r_{3}\right)$ from the nodule's centre:

$$
\mathrm{d} n_{C 2}\left(r_{3}\right)=2 r_{3} N_{3} f_{3}\left(r_{3}\right) \mathrm{d} r_{3}
$$

The distance from the centre is double, because the nodule can be cut above or below its middle.

In turn, among the class $C 2$ the mark radius $r_{2}$ will meet the inequality $r_{2} \geq x$, only if a particle centre lies closer to the cutting plane, on both sides of it, than the distance $\sqrt{r_{3}^{2}+x^{2}}$. These particles establish a new class $C 1$ and its quantity $\mathrm{d} n_{C 1}$ can by calculated by multiplying Equation (14) by the probability (12):

$$
\mathrm{d} n_{C 1}\left(r_{3}, x\right)=2 \sqrt{r_{3}^{2}-x^{2}} N_{3} f_{3}\left(r_{3}\right) \mathrm{d} r_{3}
$$

If we integrate Equation (15) with respect to the variable $r_{3}$ between the limit from $x$ to $R_{\max }$, we will get the total nodule count $N_{2}(x)$ with the mark's radii $r_{2} \geq x$ :

$$
N_{2}(x)=2 N_{3} \int_{x}^{R_{\max }} \sqrt{r_{3}^{2}-x^{2}} f_{3}\left(r_{3}\right) \mathrm{d} r_{3}
$$


Whereas the total count $N_{2}$ of all intersected nodules visible on the cutting plane can be determined by integrating Equation (13) with respect to $r_{3}$ between the limit from 0 to $R_{\max }$. Notice that the same value of $N_{2}$ can be obtained by substituting 0 instead of $x$ in the integral in Equation (16):

$$
N_{2}=2 N_{3} \int_{0}^{R_{\max }} r_{3} f_{3}\left(r_{3}\right) \mathrm{d} r_{3}
$$

Dividing Equation (16) by Equation (17) we obtain the fraction of all visible marks on a section with the radii $r_{2} \geq x$ :

$$
1-F_{2}(x)=\frac{\int_{x}^{R_{\max }} \sqrt{r_{3}^{2}-x^{2}} f_{3}\left(r_{3}\right) \mathrm{d} r_{3}}{\int_{0}^{R_{\max }} r_{3} f_{3}\left(r_{3}\right) \mathrm{d} r_{3}}
$$

The left hand side of Equation (18) results from the fact that any function $F_{X}(x)$ is defined for a random variable $X$ that takes a value of $X \leq x$ (recall Eq. (2)) and it returns outcomes from the range from 0 to 1 . In turn, Equation (18) is defined for a random radius $r_{2} \geq x$ and it yields results that belong to the range from $r_{2}$ to 1 .

The denominator in Equation (18) has the form of the expected value $E\left[r_{3}\right]$. This equation can be reformulated to obtain:

$$
F_{2}(x)=\frac{E\left[r_{3}\right]-\int_{x}^{R_{\max }} \sqrt{r_{3}^{2}-x^{2}} f_{3}\left(r_{3}\right) \mathrm{d} r_{3}}{E\left[r_{3}\right]}
$$

We can differentiate Equation (19) with respect to $x$ in order to obtain the $\mathrm{PDF}_{2}$ (see the dependency in Equation (9)). To calculate the derivative of the integral in the numerator we must use Leibniz integral rule. According to this rule, the derivative is equal to:

$$
\frac{\mathrm{d}}{\mathrm{d} x}\left(\int_{x}^{R_{\max }} \sqrt{r_{3}^{2}-x^{2}} f_{3}\left(r_{3}\right) \mathrm{d} r_{3}\right)=-\int_{x}^{R_{\max }} \frac{x}{\sqrt{r_{3}^{2}-x^{2}}} f_{3}\left(r_{3}\right) \mathrm{d} r_{3}
$$

Substituting Equation (20) into differentiated Equation (19) we get the final formula:

$$
f_{2}(x)=\frac{x}{E\left[r_{3}\right]} \int_{x}^{R_{\max }} \frac{f_{3}\left(r_{3}\right)}{\sqrt{r_{3}^{2}-x^{2}}} \mathrm{~d} r_{3}
$$

An analysis of the above equation will be presented in Section 5.

\section{LINEAR ANALYSIS}

The following relation $r_{3}^{2}=r_{1}^{2}+h_{1}^{2}$ links the particle's radius $r_{3}$ and the half chord's length $r_{1}$ located on the random cutting plane (Fig. 2). The variable $h_{1}$ is the distance between the particle's centre and the chord. Nodules with radii $r_{3}$ will have half chords' length $r_{1}$, meeting the inequality $2 r_{1} \geq 2 x$, under two conditions, Equation (10) and:

$$
h_{1} \leq \sqrt{r_{3}^{2}-x^{2}}
$$

To calculate the probability of meeting the condition (22) we can use the definition from Equation (8). In order to understand this better, let us depict Figure 2 from a different perspective so that the chord is perpendicular to the picture as it is seen in Figure 3. The length $h_{1}$ fulfils the condition (22), if the random chord pierces the nodule somewhere on the hatched area. Therefore, the probability of this event is equal to the ratio of the hatched area to the area of the nodule's projection:

$$
\operatorname{Pr}\left(h_{1} \leq \sqrt{r_{3}^{2}-x^{2}}\right)=\frac{r_{3}^{2}-x^{2}}{r_{3}^{2}}
$$

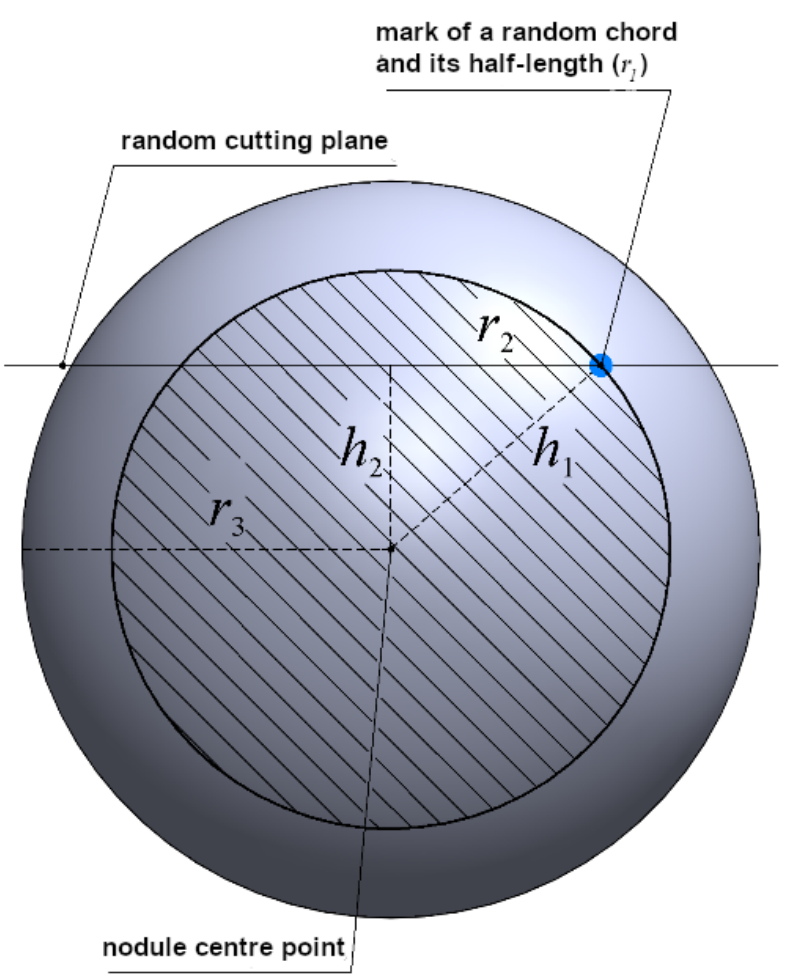

Fig. 3. Spherical particle pierced by a random chord. The direction of the chord is perpendicular to the picture and pierces the nodule in the blue dot

In order to continue our analysis, we need to introduce three nodule classes, as we did in the previous section. The sense of the first class $C 3$ has not been changed. The set $C 3$ contains these nodules that have their radii in the range from $r_{3}$ to $r_{3}+\mathrm{d} r_{3}$. The count of the particles belonging to this set can be calculated by Equation (13). If we place a random secant in the sample space (Fig. 1), generally this line will pierce only some nodules from the set $C 3$. These pierced particles will establish a new set denoted $C$ '2. The element quantity $\mathrm{d} n_{C^{\prime},}$, per unit length of this line, of the $C^{\prime} 2$ can be calculated by multiplying Equation (13) by the area of the nodule's projection:

$$
\mathrm{d} n_{C^{\prime} 2}\left(r_{3}\right)=\pi r_{3}^{2} N_{3} f_{3}\left(r_{3}\right) \mathrm{d} r_{3}
$$


Among the class $C^{\prime} 2$ the half chords $r_{1}$ meeting the inequality $2 r_{1} \geq 2 x$ will establish a subset of $C^{\prime} 2$ denoted $C^{\prime} 1$. The nodule count $\mathrm{d} n_{C^{\prime}}$, per unit length, is calculated by multiplying probability (23) by Equation (24):

$$
\mathrm{d} n_{C^{\prime} 1}\left(r_{3}, x\right)=\pi\left(r_{3}^{2}-x^{2}\right) N_{3} f_{3}\left(r_{3}\right) \mathrm{d} r_{3}
$$

The total quantity $N_{1}(x)$ of all half chords meeting the condition $2 r_{1} \geq 2 x$, for all pierced nodules, can be yielded by integrating Equation (25) with respect to the variable $r_{3}$ between the limit from $x$ to $R_{\max }$ :

$$
N_{1}(x)=\pi N_{3} \int_{x}^{R_{\max }}\left(r_{3}^{2}-x^{2}\right) f_{3}\left(r_{3}\right) \mathrm{d} r_{3}
$$

The count $N_{1}$ of all visible chords on a random cutting plane can be determined by integrating Equation (24) with respect to $r_{3}$ between the range from 0 to $R_{\max }$ :

$$
N_{1}=\pi N_{3} \int_{0}^{R_{\max }} r_{3}^{2} f_{3}\left(r_{3}\right) \mathrm{d} r_{3}
$$

The same value of $N_{1}$ can be obtained in Equation (26) by substituting 0 instead of $x$.

If we divide Equation (26) by Equation (27), we get the fraction of the half chords $r_{1}$ that meet the inequality $2 r_{1} \geq 2 x$ :

$$
1-F_{1}(x)=\frac{\int_{x}^{R_{\max }}\left(r_{3}^{2}-x^{2}\right) f_{3}\left(r_{3}\right) \mathrm{d} r_{3}}{\int_{0}^{R_{\max }} r_{3}^{2} f_{3}\left(r_{3}\right) \mathrm{d} r_{3}}
$$

The left hand side of Equation (28) can be explained in a similar manner as is done for Equation (18).

Multiplying the numerator and the denominator in Equation (28) by the constant $4 \pi$ and after rearranging this equation, we obtain the following formula:

$$
F_{1}(x)=\frac{\bar{S}-4 \pi \int_{x}^{R_{\max }}\left(r_{3}^{2}-x^{2}\right) f_{3}\left(r_{3}\right) \mathrm{d} r_{3}}{\bar{S}}
$$

where:

$$
\bar{S}=E\left[S_{3}\right]=4 \pi \int_{0}^{R_{\max }} r_{3}^{2} f_{3}\left(r_{3}\right) \mathrm{d} r_{3}
$$

is the expected value of the particles' outer area.

If we differentiate Equation (29) with respect to $x$, we obtain the $\mathrm{PDF}_{1}$ (see the dependency in Eq. (9)). To calculate the derivative of the integral in the numerator, we will use the Leibnitz integral rule. According to this rule, the derivative is equal to:

$$
\frac{\mathrm{d}}{\mathrm{d} x}\left(\int_{x}^{R_{\max }}\left(r_{3}^{2}-x^{2}\right) f_{3}\left(r_{3}\right) \mathrm{d} r_{3}\right)=-2 x \int_{x}^{R_{\max }} f_{3}\left(r_{3}\right) \mathrm{d} r_{3}
$$

Equation (31) can now be substituted into differentiated Equation (29). After reformulating, we obtain the form of the $\mathrm{PDF}_{1}$ :

$$
f_{1}(x)=\frac{8 \pi x}{\bar{S}} \int_{x}^{R_{\max }} f_{3}\left(r_{3}\right) \mathrm{d} r_{3}
$$

Applying the definitions from Equations (1) and (5) to the integral in Equation (32) we can transform this equation into the following form:

$$
f_{1}(x)=\frac{8 \pi x}{\bar{S}}\left(F_{3}\left(R_{\max }\right)-F_{3}(x)\right)
$$

Because all random chords have their length less than or equal to $R_{\text {max }}$, we can write $F_{3}\left(R_{\max }\right)=1$ and therefore:

$$
f_{1}(x)=\frac{8 \pi x}{\bar{S}}\left(1-F_{3}(x)\right)
$$

Hence, rearranging the above equation yields:

$$
F_{3}(x)=1-\frac{\bar{S}}{8 \pi x} f_{1}(x)
$$

Equation (35) allows us to estimate the form of an unknown function $\mathrm{CDF}_{3}$ on the basis of the empirically obtained function $\mathrm{PDF}_{1}$. Calculating derivatives on both sides with respect to $x$, we get the final dependency:

$$
f_{3}(x)=\frac{\bar{S}}{8 \pi}\left(\frac{f_{1}(x)}{x^{2}}-\frac{1}{x} \frac{\mathrm{d} f_{1}(x)}{\mathrm{d} x}\right)
$$

Notice, that in this formula there is an unknown parameter $\bar{S}$ on the right side. In the next section of this paper, how to estimate the value of this parameter will be explained.

The derived formula (36) is similar to known solutions proposed by Cahn and Fullman [5], Lord and Willis [6], and Spektor [7]:

$$
N_{3} f_{3}(x)=\frac{N_{1}}{2 \pi}\left(\frac{f_{1}(x)}{x^{2}}-\frac{1}{x} \frac{\mathrm{d} f_{1}(x)}{\mathrm{d} x}\right)
$$

\section{ANALYSIS OF THE OBTAINED EQUATIONS}

In the derived Equation (21), the sought function $f_{3}\left(r_{3}\right)$ is under the integral. It means that the equation cannot be transformed into an explicit form. Another fact is that it also contains an additional unknown parameter which is the expected value $E\left[r_{3}\right]$. Generally, this equation is well known and has been used and analysed many times by various authors in papers $[13,14]$. In [14] there are presented, in a form of a short description, methods used for solving this equation and how to estimate the value of $E\left[r_{3}\right]$.

The second obtained Equation (36) includes the function $f_{3}(x)$ expressed in an explicit form. However, in order to use it in practice, it must be transformed into a discrete form. 
The data, i.e. a set of chords, creates the function $f_{1}(x)$ in a form of a histogram which is not a continuous function. But, in a computer image analysis this is not a big problem. We can get a large enough collection of chords to estimate the function $f_{1}(x)$ with practically any small step and hence to produce the function $f_{3}(x)$ with satisfactory accuracy. Equation (36) includes also an unknown value of $\bar{S}$. We will propose now how to estimate this value.

Let us integrate both sides of Equation (36) in the range from 0 to $R_{\max }$ :

$$
\int_{0}^{R_{\max }} f_{3}(x) \mathrm{d} x=\frac{\bar{S}}{8 \pi} \int_{0}^{R_{\max }}\left(\frac{f_{1}(x)}{x^{2}}-\frac{1}{x} \frac{\mathrm{d} f_{1}(x)}{\mathrm{d} x}\right) \mathrm{d} x
$$

According to Equation (4) the left hand side integral of the above formula is equal to 1 . After rearranging we get an equation that allows us to estimate the value of $\bar{S}$ on the basis of experimental data:

$$
\bar{S}=\frac{8 \pi}{\int_{0}^{R_{\max }}\left(\frac{f_{1}(x)}{x^{2}}+\frac{1}{x} \frac{\mathrm{d} f_{1}(x)}{\mathrm{d} x}\right) \mathrm{d} x}
$$

An analysis of Equation (34) allows us to detect intervals in which there are no nodules with the radius belonging to those ranges. Suppose, we have a range from $x_{1}$ to $x_{2}$. If this range does not contain any radius $r_{3}$, this means that the function $F_{3}(x)$ has a constant value. This fact concludes that the function $f_{1}(x)$ depends proportionally on the variable $x$, i.e. $f_{1}(x)=c \cdot x$. The proportional coefficient $c$ is equal:

$$
c=\frac{8 \pi}{\bar{S}}\left(1-F_{3}(x)\right)
$$

The above coefficient has a sense of a tangent of the angle between the $X$ axis and the proportional range of the function $f_{1}(x)$. This tells us that not only each interval with no particles is proportional, but also its direction passes through the value of 0 on the $X$ axis.

Let us consider a particular situation when the sample space is bounded from the bottom - it contains nodules with a minimal radius $R_{\min }$. An example of such a sample is depicted in Figure 4 and corresponding histograms of PDF and $\mathrm{PDF}_{3}$ are shown in Figure 5.

Notice, even if in the sample there are no nodules with a radius of less than $R_{\text {min }}$, the collected data contains chords with a length less than the value of $R_{\text {min }}$. The shorter chords can be obtained not only by piercing the minimal particles, but also from larger nodules that are pierced by a random secant at a large enough distance from their centres.

As it is schematically presented in Figure $5 \mathrm{~b}$ the $f_{3}(x)$ is equal to 0 for $x<R_{\min }$. The zero value of the $f_{3}(x)$ also results a zero value of its $F_{3}(x)$. Because of this reason, the function (34) and its histogram have a proportional character in a range from 0 to $x_{a}$ :

$$
f_{1}(x)=\frac{8 \pi}{\bar{S}} x
$$

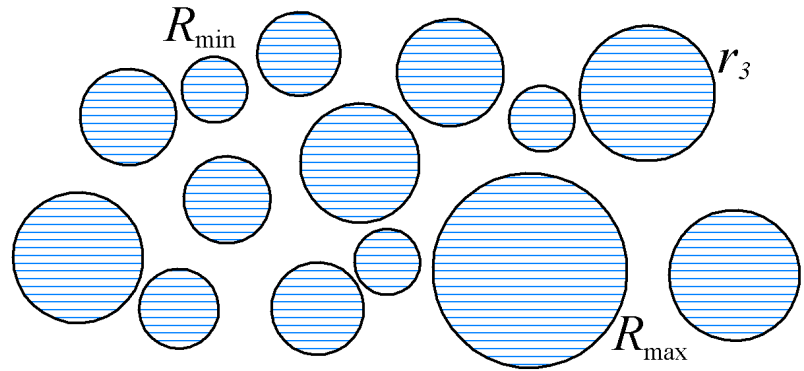

Fig. 4. Set of chords (blue lines) obtained from a sample that contains nodules with a minimal radius $R_{\min }$

a)

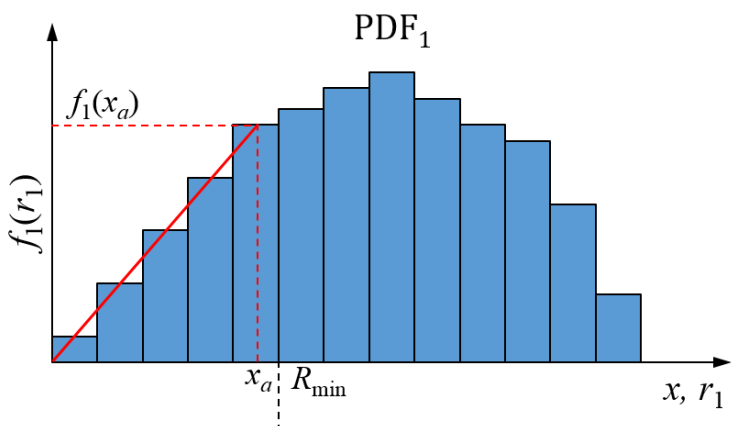

b)

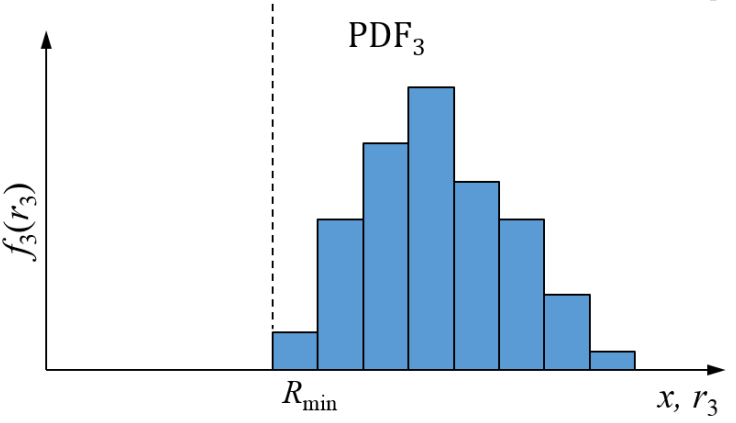

Fig. 5. Scheme presenting how to graphically determine the value of $x$ needed to estimate the unknown parameter: a) a histogram of a chord size distribution; b) a histogram of a nodule size distribution

This fact allows us to state that if the beginning of a histogram of the $\mathrm{PDF}_{1}$ is proportional, this range does not contain particles with this size because the $F_{3}(x)$ is zero. The proportional dependency can be determined graphically as it is presented on the histogram in Figure 5a. In this case, having the values of $x_{a}$ and $f_{1}\left(x_{a}\right)$ we can use a simpler formula to estimate the unknown $\bar{S}$ by substituting these values to Equation (41) in order to get:

$$
\bar{S}=\frac{8 \pi}{f_{1}\left(x_{a}\right)} x_{a}
$$

Further analysis of Equation (34) permits us to extend the property described in the previous paragraph. This means that if any range of the $f_{1}(x)$ histogram has a proportional dependency, the function $F_{3}(x)$ must be constant in this range to fulfil this proportionality. In turn, according to the obvious relation in Equation (6) the function $f_{3}(x)$ takes the value of 0 everywhere where $F_{3}(x)$ is constant. This allows us to claim that each proportional scope of the $\mathrm{PDF}_{1}$ has no nodules with radii which belong to this range. 


\section{CONCLUSIONS}

The presented research in this paper can be concluded in the following points:

- Two derived Equations (21) and (35) use sets of outcomes obtained experimentally as input data. The data is collected from a two-dimensional cross section and has a form of sets containing mark radii and random chords.

- The obtained equation, which uses a set of mark radii, has the same form as the form derived by other authors. For this reason, this equation was not analysed further in the paper.

- Having experimentally obtained a set of chords, the derived Equation (38) allows us to reveal an unknown shape of a function that describes the PDF3 of three-dimensional particle radii.

- Detection of any proportional dependency on a PDF1 histogram allows us to state that the sample space does not contain particles with radii that belong to that proportional scope. This property results directly from the analysis of Equation (34).

- The most valuable thing in the presented derivation is Equation (34). It allows stating the absence of particles with certain radii. This fact cannot be concluded from Equation (37) derived by other authors, even though Equation (37) can also produce histograms with proportional ranges.

- Both equations, the derived one (36) as well as the proposed by other authors (Eq. (37)) require determining parameters ( $\bar{S}$ and $N_{3}$, respectively) from 3D space having only outcomes from 2D cross sections. We proposed two manners how to estimate the value of by using Equations (39) and (42).

\section{REFERENCES}

[1] Wicksell S.D. (1925). The Corpuscle Problem: A Mathematical Study of a Biometric Problem. Biometric, 17(1-2), 84-89.

[2] Sheil E. (1935). Statistische Gefügeuntersuchungen I. Zeitschrift für Metallkunde, 27(9), 199-208.
[3] Schwartz H.A. (1934). The Metallographic Determination of the Size Distribution of Temper Carbon Nodules. Metals and Alloys, $5,139-140$.

[4] Saltykov S.A. (1967). The Determination of the Size Distribution of Particles in an Opaque Material from the Measurement of the Size Distribution of their Section. In: The Second International Congress for Stereology, Chicago, 8-13 April 1967. Berlin - Heidelberg - New York, Springer Verlag.

[5] Cahn J.W. \& Fulmann R.L. (1956). On the Use of Lineal Analysis for Obtaining Particle Size Distributions in Opaque Samples. Transactions of the American Institute of Mining, Metallurgical and Petroleum Engineers, 206, 177-187.

[6] Lord G.W. \& Willis T.F. (1951). Calculation of Air Bubble Size Distribution from Results of a Rosiwal Traverse of Aerated Concrete. ASTM Bulletin, 177, 177-187.

[7] Спектор А. Г. (1950). Дисперсионный анализ сферических частиц в непрозрачных структурах. Заводская лаборатория. Диагностика материалов. 16.(2), 173-177. [Spektor A.G. (1950). Dispersionnyy analiz sfericheskikh chastits v neprozrachnykh strukturakh. Zavodskaya laboratoriya. Diagnostika, Materialov, 16(2), 173-177].

[8] Hakahashi J. \& Suito H. (2003). Evaluation of the Accuracy of the Three-Dimensional Size Distribution Estimated from the Schwartz-Saltykov Method. Metallurgical and Materials Transactions, 34A, 171-181.

[9] Pedersen K.M. \& Tiedje N.S. (2008). Graphite nodule count and size distribution in thin-walled ductile cast iron. Materials Characterization, 59, 1111-1121.

[10] Mrzygłód B., Matusiewicz P., Tchórz A. \& Olejarczyk-Wożeńska I. (2013). Quantitative Analysis of Ductile Iron Microstructure A Comparison of Selected Methods for Assessment. Archives of Foundry Engineering, 13(3), 59-63.

[11] Basak C.B. \& Sengupta A.K. (2004). Development of a FDM based code to determine the 3-D size distribution of homogeneously dispersed spherical second phase from microstructure: a case study on nodular cast iron. Scripta Materialia, 51, 255-260.

[12] Gurgul D., Burbelko A. \& Wiktor T. (2018). Analysis of Spherical Particle Size Distribution - Theoretical Basis. Archives of Foundry Engineering, 18(1), 29-34.

[13] Li T., Shimasaki S., Taniguchi S. \& Narita S. (2016). Reliability of Inclusion Statistics in Steel Stereological Methods. ISIJ International, 56(9), 1625-1633.

[14] Wiencek K., Skowronek T. \& Khatemi B. (2005). Graphite Particle Size Distribution in Nodular Cast Iron. Metallurgy and Foundry Engineering, 31(2), 167-173. 


\section{LIST OF VARIABLES USED IN THE TEXT}

c - proportional coefficient,

$C 1$ - subclass of the $C 2$ containing only these particles that have their mark radii $r_{2} \geq x$,

$C^{\prime} 1$ - subclass of the $C^{\prime} 2$ containing only these particles that have their half-length chord $2 r_{1} \geq 2 x$,

$C 2$ - subclass of the $C 3$ containing only these particles that are split by the random cutting plane,

$C 2$ - subclass of the $C 3$ containing only these particles that are pierced by a random secant,

C3 - class containing all the particles that have their radius in the range from $r_{3}$ to $r_{3}+\mathrm{d} r_{3}$,

$\mathrm{d} n_{C 1}$ - quantity, per unit area, of the particles belonging to the $C 1$,

$\mathrm{d} n_{C^{\prime} 1}-$ quantity, per unit length, of the particles belonging to the $C^{\prime} 1$,

$\mathrm{d} n_{C 2}-$ quantity, per unit area, of the particles belonging to the $C 2$,

$\mathrm{d} n_{C 2}$ - quantity, per unit length, of the particles belonging to the $C^{\prime}$ ',

$\mathrm{d} n_{C 3}-$ quantity, per unit volume, of the particles belonging to the $C 3$,

$F_{1}(x)$ - Cumulative Distribution Function $\left(\mathrm{CDF}_{1}\right)$ of a halflength of a random chord $r_{1}$,

$F_{2}(x)$ - Cumulative Distribution Function $\left(\mathrm{CDF}_{2}\right)$ of a random mark radius $r_{2}$
$F_{3}(x)$ - Cumulative Distribution Function $\left(\mathrm{CDF}_{3}\right)$ of a random nodule radius $r_{3}$,

$f_{1}(x)$ - Probability Density Function $\left(\mathrm{PDF}_{1}\right)$ of a half-length of a random chord $r_{1}$,

$f_{2}(x)$ - Probability Density Function $\left(\mathrm{PDF}_{2}\right)$ of a random mark radius $r_{2}$,

$f_{3}(x)$ - Probability Density Function $\left(\mathrm{PDF}_{3}\right)$ of a random nodule radius $r_{3}$,

$h_{1}$ - distance between a nodule centre and a random chord,

$h_{2}$ - distance between a nodule centre and a random cutting plane,

$N_{1}$ - quantity, per unit length, of all particles pierced by a random secant,

$N_{2}$ - quantity, per unit area, of all particles intersected by a random cutting plane,

$N_{1}(x)$ - the total particle count with the half chord $2 r_{1} \geq 2 x$,

$N_{2}(x)$ - the total particle count with the mark radii $r_{2} \geq x$,

$N_{3}$ - volumetric particle density (a mean nodule count per unit volume),

$R_{\max }$ - the largest radius of a nodule in the sample space,

$R_{\min }$ - the smallest radius of a nodule in the sample space,

$r_{1}$ - half-length of a random chord,

$r_{2}$ - radius of a nodule section made by a random cutting plane,

$r_{3}$ - radius of a random nodule,

$\frac{3}{S}$ - the expected value of the outer particles' area,

$x$ - real number or a variable used in equations,

$x_{a}$ - value up to which the function $f_{3}(x)$ is proportional. 